\title{
EL DESARROLLO SUSTENTABLE DESDE UNA MIRADA UNIVERSITARIA INTERCULTURAL
}

\author{
SUSTAINABLE DEVELOPMENT FROM AN INTERCULTURAL \\ UNIVERSITY PERSPECTIVE
}

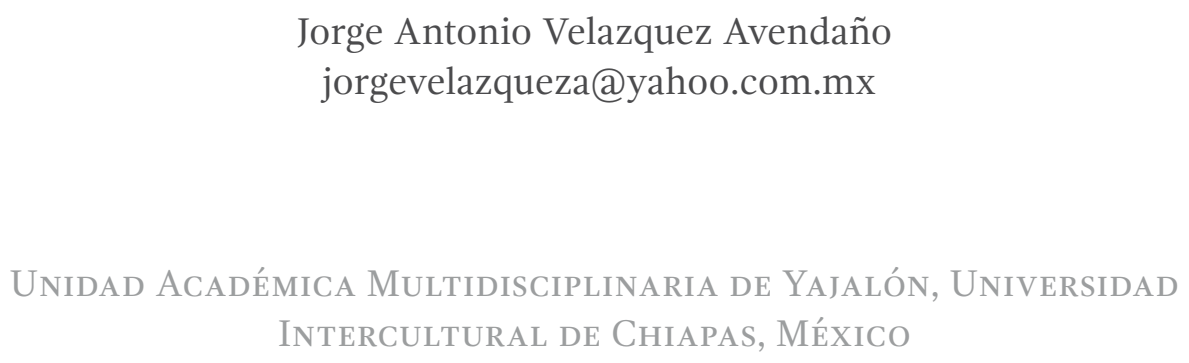


Para citar este artículo:

Velazquez Avendaño, J. A. (2020). El Desarrollo Sustentable desde una mirada universitaria Intercultural. ESPACIO I+D, INNOVACIÓN MÁS DESARROLLO, 9(24). https://doi.org/10.31644/ IMASD.24.2020.a07

\section{RESUMEN}

El encuentro de conceptos, que suscitan mucho interés y grandes expectativas suelen, también promover la reflexión y el análisis en la búsqueda de definiciones que contribuyan a entender mejor sus significados, como es el caso de los términos sustentabilidad, desarrollo, interculturalidad, y vinculación comunitaria, en particular en contextos interculturales, es decir, en espacios donde convergen comunidades que, se supone, son diferentes, como las indígenas y mestizas, y que terminan por enriquecer el proceso reflexivo. Mucha intención ha corrido en torno al concepto Sustentabilidad, pero poco ha transcurrido si lo asociamos al concepto de Desarrollo e Interculturalidad, una comunión que permite echar una mirada al desarrollo de las comunidades desde una perspectiva diferente, de la mirada de la gente en torno a su diario vivir bajo un enfoque sustentable, de su percepción de cosas tan obvias del día a día como es el manejo de la basura en sus casas hasta problemas complejos como la contaminación de ríos y cuencas por las acciones humanas; es preguntarnos cómo quiere vivir la gente y cómo aplica la sustentabilidad en su entorno y de cómo se relaciona con otros grupos culturales diferentes, pero que comparten un mismo territorio. En este sentido, la Sustentabilidad, el Desarrollo y la Interculturalidad promueven la necesidad de la vinculación a las comunidades que proceden de culturas diferentes, y se convierten en las razones universitarias interculturales para movilizarse, son sus motivos de existir, cuyo propósito es descubrir sus pensamientos y sus acciones en torno a esta cuestión del Desarrollo Sustentable. La pretensión de este trabajo es precisamente contribuir a la reflexión y análisis acerca del concepto Desarrollo Sustentable en un contexto de Interculturalidad, cuya importancia está en que diversas universidades, en particular las interculturales, lo promueven como una carrera profesional ligada íntimamente a un eje pedagógico central que es reconocido como vinculación comunitaria, el cual es visto como un fundamento formativo de especial importancia para sus profesionales en formación en Desarrollo Sustentable, el cual facilita, entre otros atributos, el reconocer y explicar el impacto que en la calidad de vida de las comunidades humanas tiene el uso y preservación de los recursos (entendido como naturales, humanos, ecológicos, etc.), en la actualidad y predecir su efecto en el futuro.

Palabras clave

Sustentabilidad, Desarrollo, Interculturalidad, Vinculación comunitaria. 
The meeting of concepts that arouse much interest and high expectations also usually promote reflection and analysis in the search for definitions that contribute to a better understanding of their meanings, such as the terms sustainability, development, interculturality, and community bonding, particularly in intercultural contexts, that is, in spaces where communities that are supposed to converge are different, such as indigenous and mestizo, and that end up enriching the reflexive process. A lot of intention has run around the concept of Sustainability, but little has happened if we associate it with the concept of Development and Interculturality, a communion that allows you to take a look at the development of communities from a different perspective, from the look of people around his daily live under a sustainable approach, from his perception of things as obvious from day to day as is the handling of garbage in their homes to complex problems such as the contamination of rivers and basins by human actions; It is to ask ourselves how people want to live and how they apply sustainability in their environment and how it relates to other different cultural groups, but that share the same territory. In this sense, Sustainability, Development and Interculturality promote the need of linking to communities that come from different cultures, and become the intercultural university reasons to mobilize, are their reasons for existing, whose purpose is to discover their thoughts and its actions around this issue of Sustainable Development. The aim of this work is precisely to contribute to the reflection and analysis of the concept of Sustainable Development in a context of Interculturality, whose importance is that various universities, particularly intercultural ones, promote it as a professional career closely linked to a central pedagogical axis which is recognized as community bonding, which is seen as a training foundation of special importance for its professionals in training in Sustainable Development, which facilitates, among other attributes, recognizing and explaining the impact on the quality of life of Human communities have the use and preservation of resources (understood as natural, human, ecological, etc.), at present and predict their effect in the future.

\section{Keywords}

Sustainable, Development, Interculturality, Community engagement. 
$\mathrm{E}$ n el devenir del desarrollo o progreso de la humanidad hacia mejores condiciones de bienestar, uno de los ejes centrales en este dominio son los llamados "paradigmas del desarrollo" que al finalizar la segunda guerra mundial tienen un impulso sin precedentes, pero sin una propuesta de fondo, ya que privilegia el uso de los recursos naturales en aras del crecimiento económico, el cual se suponía "ilimitado". Sin embargo, no es hasta la década de los 80 del siglo XX, en que irrumpe en el escenario un elemento conocido como Sustentabilidad, que pone en entredicho y en la mesa de análisis "el crecimiento ilimitado" y se convierte en una especie de espejo de medición de estos paradigmas y sus políticas puestas en marcha. Este concepto, hasta ese momento no muy conocido, llama la atención mundial al exponer los riesgos para la continuidad, ya no solo del progreso humano, sino la de su existencia misma como consecuencia o efecto de esas políticas de desarrollo implementadas, incluso hasta el día de hoy, cuyos efectos impactan negativamente en el ambiente.

Muchas naciones fincaron su desarrollo en estos modelos, dictados desde los centros de poder mundial y que indudablemente trajeron consigo progreso, pero también consecuencias que la Comisión Brundtland de la Organización de las Naciones Unidas (ONU, 1987) planteó, dando cuenta de que el bienestar humano, su desarrollo social y económico, pero también su sobrevivencia, dependen del uso racional y la preservación de los recursos naturales ( $\mathrm{RN}$ ) para las generaciones futuras, colocándolo como un factor medular de medición del desarrollo y por lo tanto de la elaboración de nuevas políticas públicas que contemplen el desarrollo de las naciones desde una perspectiva del desarrollo integral a partir del trinomio ecológico-económico-social.

Tal premisa trae consigo retos que convocan a profundizar en el estudio y análisis del concepto Desarrollo Sustentable, desde las diferentes perspectivas posibles, en especial desde la universitaria intercultural pues el motivo que las anima es la comprensión, tanto de las visiones como de las interrelaciones de las comunidades humanas culturalmente diferentes, que convergen en sitios o territorios comunes, por medio de acciones que se proponen descubrir los elementos o componentes que subyacen en estos territorios pluri e interculturales, con el supremo propósito de formar profesionales en esta área que contribuyan a entender mejor el desarrollo local desde un enfoque sustentable.

\section{DE LA INTERCULTURALIDAD Y SU CORRESPONDENCIA CON EL DESARROLLO SUSTENTABLE}

Un elemento de mucho interés para el estudio del Desarrollo Sustentable, desde las comunidades que son culturalmente diversas, pero que con- 
viven y comparten territorios comunes, es el término reconocido como Interculturalidad cuya comprensión ha sido tratado por diversos autores como C. Walsh (2007), que la explica desde tres perspectivas que reconoce como relacional, funcional y crítica, mientras otros como Albo (2003), la sitúan a partir de tres cuestiones importantes: (1) Identidad de personas que comparten una cultura específica, (2) Diferenciación de otros grupos de diferente cultura, y (3) Correspondencia de los grupos que comparten elementos culturales y que hacen posible la conexión en la diversidad cultural.

Como quiera que sea, es muy importante tener presente que cuando hablamos de Interculturalidad hacemos referencia a un concepto que implica cambios a través del tiempo y que puede ser estático o dinámico, a lo que nos referiremos un poco más adelante.

La interculturalidad, en una primera aproximación, podemos observar que se nutre de otro concepto cuya característica principal es su complejidad, nos referimos al término de cultura, el cual, cuando menos, agrupa tres cuestiones relevantes: 1.- las relaciones con la naturaleza, el desarrollo material y económico (producción, alimento, vivienda, etc.); 2.- las relaciones sociales entre personas y grupos (familia, comunidad, política); y 3.- la imaginaria (o simbólica) que da sentido al conjunto: lenguaje, religión, arte, sistema jurídico y legal (Albo, 2003).

Si a este término de cultura agregamos el sufijo inter podemos observar dos cuestiones de interés, por un lado, este sufijo tiene una connotación de movimiento, de dinamismo, como fluidos que van y vienen en una conexión intensa; y por otro, coloca un sentido de existencia de, cuando menos, dos polos y hace referencia así al reconocimiento de que cuando menos hay una conexión, una relación, de dos culturas cuyos fluidos se dirigen de un lado al otro y viceversa.

En los hechos no solo se conectan o relacionan dos polos sino muchos polos. Y tal situación convierte al término interculturalidad de la mayor relevancia para entender las relaciones entre las diferentes culturas del mundo. Al menos este término, tiene tres aspectos que cabe destacar:

El primero es el dinamismo presente en la interculturalidad. Debe entenderse como una relación profunda de al menos dos culturas, que tienen un impacto generalizado, particularmente en las conductas (nos referimos a las formas visibles en que se conducen los seres humanos), sobre todo, en el carácter del hombre (nos referimos a aquello no visible, pero que es el motivo real de la conducta del hombre), es decir, los cambios en la caracterología de los seres humanos (Fromm, 2006), que al entrar en contacto se influyen mutuamente, considerando que este contacto puede ser violento y de imposición o sutil y amigable; como sea, el impacto provoca cambios sustanciales en todos los aspectos, desde el carácter hasta las manifestaciones 
culturales, y es lo que debería de tener mayor énfasis en los estudios que se realizan desde la interculturalidad.

El segundo es el contacto e intercambio estático. Podría pensarse que no tiene mayores implicaciones en la caracterología del hombre y en las relaciones sociales; no obstante, aun cuando los cambios no son considerados de relevancia para las culturas que están interconectadas, no dejan de tener su importancia en la modificación de ciertos patrones de conducta y de algunos cambios de perspectivas y/o cosmovisiones, lo que puede conducirlas a una suerte de lo que Albo (2003), llama inculturación, o derivar en alguna otra cómo la transculturación, etc.

Finalmente tenemos un aspecto muy importante a tener en cuenta, el análisis histórico de las culturas comprometidas (Harris, 1986; Quijano, 2000), tanto de los grupos sociales involucrados y sus rasgos caracterológicos predominantes cómo del encuentro mismo, ya que a partir del análisis histórico de esos encuentros culturales encontramos elementos para entender el devenir actual del comportamiento de nuestras interculturalidades. Como ejemplo muy cercano a nuestra América Latina, podría bastar el encuentro de dos culturas diametralmente opuestas: la cultura española y las mesoamericanas.

A la luz de ese encuentro, es posible observar, desde una perspectiva intercultural, una dicotomía que podemos analizar desde los papeles de subyugador-sometido, dado en un marco de encuentro tanto violento y brutal, como de astucia y marrullería, que dan como resultado el sometimiento y despojo de una cultura por la otra, operada por medio de la introducción de un nuevo elemento o instrumento de sumisión y poder reconocido como "raza" (Quijano, 2000), que llevó a la estructuración de una sociedad de "razas" y de "castas" con todas las características de sometimiento y explotación de la cultura dominante. Esta pervive, de una u otra manera, hasta nuestros días, que bien puede ser identificada como la Colonialidad del poder (Walsh, 2006), que describe toda una cultura de dominación heredada a través de los siglos.

Considerando lo mencionado y a partir de nuestras observaciones y experiencias en el devenir del tiempo, en la Universidad Intercultural de Chiapas, específicamente en la Unidad Académica Multidisciplinaria de Yajalón, desde una perspectiva de formación-educación escolar, ha sido posible visualizar, en un primer acercamiento a los grupos culturales que en ella participan, una doble relación en la construcción de las relaciones interculturales, entendida como lo dicen Baronnet y Bermúdez (2019), como una construcción que no se da en un vacío, “...sino en medio de tensiones, conflictos y apropiaciones, inserta en relaciones sociales y de poder, y configurada por la historia del contexto regional...", este es un efecto positivo que va más allá de las conversaciones e interacciones de los lazos de amistad 
y convivencia entre estudiantes que provienen de culturas diferente, bien sea indígenas o mestizas, ya que existe un desarrollo armónico y de mutua tolerancia, además hemos registrado esa herencia histórica del racismo, el menosprecio de una cultura por la otra, bien sea Tseltal, Ch'ol, o mestiza, pero que busca la descalificación del otro.

Para el primer caso, se observa una aceptación tácita de la existencia de otras culturas diferentes a la de uno, acompañada de un interés creciente por saber más del otro, reconocer como son, como se comportan, qué actividades económico-productivas realizan, qué actividades socio-culturales llevan a cabo, cómo lo hacen, por qué lo hacen y cuáles son las acciones que emprenden para proteger el medio, que bien puede resumirse en las acciones que muchas comunidades llevan a cabo en los rituales para pedir por el agua, por ejemplo, o para las cosechas, etc., Baronnet y Bermúdez (2019), lo reconocen como el relacionamiento en el proceso de vinculación. Considero que es aquí donde el concepto del Desarrollo Sustentable y la Interculturalidad encuentran su mayor correspondencia, ya que el Desarrollo Sustentable se convierte en catalizador de valores, conductas y actitudes de las personas que en ella intervienen.

El Desarrollo Sustentable es además el portador de un acervo de conocimientos que provienen tanto de las comunidades locales como de las aulas universitarias, es decir, si bien no existe un término en las lenguas maternas que identifique y le dé significado al concepto de Desarrollo Sustentable, no impide su estudio a partir de los idiomas locales, ya que de alguna manera hay acciones de las comunidades que son identificables con el paradigma de la Sustentabilidad, de tal manera que hemos visto rituales ancestrales agro-culturales, (por ejemplo del agua), lo mismo en la ciudad de Yajalón, que es predominántemente mestiza, que en la comunidad Amado Nervo, que es Ch'ol, o que en la comunidad Aurora Grande, que es Tseltal, los estudiantes y profesores en las aulas logran abstraerlas y hacerlas converger con la filosofía que anima el planteamiento del Desarrollo Sustentable.

El estudio del segundo caso es de mucho interés para un análisis histórico desde la Interculturalidad, ya que las expresiones culturales observadas pueden ser heredadas desde el colonialismo, las que impiden, como lo dice Quijano (2000) que "todos nos sintamos ciudadanos de igual categoría”, la cual se debe visibilizar y estudiar desde el Desarrollo Sustentable, en correspondencia con la Interculturalidad. Aclarando que si bien no es la constante en el ámbito universitario intercultural, si se percibe en el territorio que nos ocupa, como lo menciona C. Walsh (2006), como expresiones de un sector dominante y opresor, heredero político y cultural de aquellos grupos conquistadores y colonizadores, y otro sector originario, subordinado, discriminado y oprimido. 
La interculturalidad, vista desde esta perspectiva. debería entonces definirse como la relación histórica entre grupos o personas de culturas diferentes, que pueden o no compartir rasgos culturales y que mantienen una interacción dinámica y/o estática de mutua influencia; por lo consiguiente, en el ámbito universitario intercultural y del Desarrollo Sustentable, las relaciones interpersonales y grupales, así como las actitudes, conductas e intercambios, son los elementos que articulan y llevan a cabo la interculturalidad.

\section{DE LA SUSTENTABILIDAD A LA FORMACIÓN DE PROFESIONALES EN DESARROLLO SUSTENTABLE}

Es de considerar que partimos de un concepto -Sustentabilidad- que ha aludido, históricamente, a diversas perspectivas e interpretaciones que han atendido diferentes escalas de aplicación y que han derivado en la formulación de una diversidad de metodologías para hacer esos reconocimientos (Sarandón y Flores, 2009), que son presentadas como confiables, oportunas y veraces desde su propia perspectiva, la cual, cabe decir, depende en buena medida tanto de la intencionalidad del estudio (entendido como el qué quiere entender, descubrir y/o conocer el investigador), como del ojo del observador y la posición en que está ubicado cuando describe la manera en que se encuentra una determinada superficie o espacio, es decir, de la posición o volumen en una determinada situación (Martínez y Figueroa, 2014).

Por lo tanto, la perspectiva es clave para entender el concepto Sustentable, lo que desemboca en la necesidad de ponderar el concepto en el contexto de las investigaciones, y también en la planificación de mapas curriculares, como es el caso del Desarrollo Sustentable, en las Universidades Interculturales, el cual debe ser abordado con sumo cuidado e interés y comprender con precisión a que se refiere.

En este contexto, en mi opinión, tenemos que reconocer que el concepto Desarrollo Sustentable contiene tres elementos clave: 1.- Nos habla del reconocimiento y análisis acerca del uso de los recursos (naturales, humanos, culturales), en el presente y su efecto en la preservación para el futuro y 2.- la calidad de vida de las comunidades humanas ahora (y también en el futuro), pero, jatención! porque son comunidades culturalmente diversas (Dietz G, 2009), participando de un mismo territorio e interrelacionándose permanentemente, influyéndose, y tal elemento introduce una situación de mayor complejidad para entender y aplicar el concepto de Desarrollo Sustentable, por lo tanto el tercer elemento central que permea en el trayecto formativo profesional es la Interculturalidad.

La relación de interés entre aquellos elementos clave y la Interculturalidad se encuentra en el efecto o impacto que se ejercen mutuamente, es decir, la exploración de la calidad de vida está conectada con la exploración en el uso 
de los recursos en un contexto cultural dado, que a su vez tiene un efecto en las relaciones que se dan entre las culturas y que derivan necesariamente en la elaboración y construcción de propuestas que deberían tomar en cuenta el contexto cultural de origen, y proponer como menciona Sarandón y Flores, (2006), un desarrollo con sustentabilidad.

En función de lo mencionado, entonces, un profesional en el Desarrollo Sustentable es aquella persona dedicada al estudio y análisis sobre como el ser humano se relaciona con su entorno, en función de la utilidad, uso y manejo de los recursos, pero también como se relaciona con otros grupos culturales diferentes, y su efecto, por un lado, en la calidad de vida de las comunidades humanas y por otro, en su impacto en el entorno natural; cuyas exploraciones integran en una primer etapa investigaciones científicas, que derivan en diagnósticos que sustentan la planificación de alternativas sustentables de solución bien sea en el diseño de proyectos de desarrollo o en planes y programas para políticas públicas.

Tal definición implica que el estudio y análisis debe tener una secuencia que inicia con la identificación y organización de datos que permitan hacer un reconocimiento del territorio y las culturas que en ella conviven, para luego hacer una descripción de componentes (estructura y función del agroecosistema) o de acontecimientos (cronología e hitos), actores sociales (¿Quién y Cómo es el sujeto social?), procesos y contextos de experiencias (Sánchez, 2010), y posteriormente, establecer su significado, es decir, sistematizar de forma crítica ¿Qué se piensa y se comprende de esa situación? ¿Qué valor y relevancia la comunidad le otorga a su realidad? ¿Cuáles son sus perspectivas de futuro? (Bonilla et al, 2009).

La secuencia conlleva al diagnóstico a la identificación de problemáticas y necesidades (Ander, 1998), a partir de la relación que se ha establecido entre comunidades humanas con una cultura específica y el uso de los recursos ¿cómo las comunidades humanas, desde la especificidad de su cultura, se han apropiado de esos recursos? ¿Cómo la cultura dominante, en esa relación intercultural, impacta en las otras y las transforma? Es necesario responder a cuestiones que, supone, reconocerán aspectos relevantes de la vida cotidiana de la comunidad (Bonilla et al, 2009), aspectos que recrean cómo es el diario vivir de las personas entre sí y su entorno, para dilucidar cómo interactúan, se comunican o se interrelacionan como grupos culturales.

Finalmente, el diagnóstico debe sustentar la planificación, dicho de otra forma, la secuencia conlleva medir el impacto que las relaciones de las comunidades con su entorno han tenido en la calidad de vida y en los recursos que llevan a la construcción de propuestas de solución y a su planificación, desde una perspectiva sustentable. 


\section{EL PROFESIONAL DEL DESARROLLO SUSTENTABLE DE LAS UNIVERSIDADES INTERCULTURALES}

El perfil del profesional en Desarrollo Sustentable de las universidades que lo impulsan, en particular las Interculturales, depende de las llamadas áreas formativas, del grupo de asignaturas insertas en los planes de estudio, que condicionan un área de especialización y esto a su vez proyecta el ámbito en el que será competente (Programa académico, 2013), por lo tanto, el perfil debería, ante todo, corresponderse con la realidad territorial donde se ubica la carrera profesional.

Esta premisa se sustenta en el hecho de que el territorio del entorno universitario tiene un fuerte impacto en la formación profesional de sus estudiantes, debido a la diversidad de culturas que en ella se encuentran y las interacciones que generan, las cuales moldean, modifican y mantienen una influencia vital en las conductas, ya que suelen fortalecer su identidad cultural, desarrollando en particular su confianza en el uso y aplicación de rasgos culturales de mucho interés como las lenguas maternas. Es aquí precisamente donde el papel que juega la vinculación comunitaria cobra mayor sentido, ya que ésta es la fuerza que anima y fomenta en sentido positivo las relaciones interculturales.

Por lo consiguiente, las asignaturas del área formativa y el peso que ejercen en los planes de formación, deberían derivar del perfil profesional que se sustenta de una realidad del territorio en cuestión y de las culturas que en ella existen, considerando sus actividades para utilizar los recursos y a las prácticas agro-eco-culturales que promueven y el efecto que estas tienen en la calidad de vida de las comunidades humanas, en particular, lo relacionado a las problemáticas y necesidades que estas desencadenan.

Por otro lado, también del perfil profesional resultan, por decirlo de alguna manera áreas "especializadas”, es decir, que ponen mayor énfasis en algunos temas de la sustentabilidad y que dependen en este sentido de las áreas formativas que se estudien, por ejemplo, puede ser un perfil profesional enfocado al componente agroecológico y de menor peso en el área industrial, por lo tanto, el tema que toca a las áreas formativas se vuelven sustanciales para alcanzar el perfil deseado en los egresados.

En este sentido, el concepto Desarrollo Sustentable implica tener en cuenta las tres dimensiones de la sustentabilidad, que debe ser la fuente que nutre las asignaturas de las áreas básicas, formativas y de especialización, aunado íntimamente al eje de vinculación comunitaria, cuya práctica fomenta los atributos y cualidades del investigador, del diagnosticador y diseñador de propuestas integrales de solución, sustentados en el respeto, responsabilidad, cuidado y conocimiento del entorno natural, principios éticos básicos para afirmar una relación de armonía. 


\section{LA VINCULACIÓN COMUNITARIA EN LA FORMACIÓN DEL PROFESIONAL DEL DESARROLLO SUSTENTABLE.}

En la formación de profesionales en el Desarrollo Sustentable, desde las Universidades Interculturales, el puente que permite conectar el desarrollo comunitario y la sustentabilidad, y a su vez la sustentabilidad con la interculturalidad, se da entre el sujeto de estudio y el investigador a través de un eje pedagógico llamado Vinculación Comunitaria, cimiento formativo de mucha importancia en el currículo profesional desde el plano normativo (Casillas y Santini, 2009), ya que conforma una línea o eje que atraviesa el plan de estudios de esta profesión, así como desde la práctica real, en la relación universidad-comunidades.

De las múltiples cualidades que este eje tiene hay dos que destacan.: La primera es que promueve la formación de competencias profesionales en la investigación científica, en la elaboración de diagnósticos comunitarios, en la planificación del desarrollo comunitario, en el diseño de proyectos de desarrollo y en la capacidad de gestión. La segunda es que promueve una formación ética, cimentada en valores que fomentan una interculturalidad basada en el respeto, la responsabilidad, el cuidado y los conocimientos. En este sentido, la vinculación comunitaria puede ser vista como una plataforma que permite realizar no solo un abordaje profundo en los sujetos de estudio, desde el punto de vista técnico, sino también como una fuerza positiva que anima a esas conexiones o relaciones, es decir, que dinamiza el proceso de las relaciones socioculturales del que participan sujetos con sus propias “...estructuras culturales, económico-políticas y relaciones de poder...” Baronnet y Bermúdez (2019), y que encuentran modos de relacionarse.

¿Cómo es en la práctica ese puente Vinculación-comunidad? Dicho de otra forma ¿Cómo se implementa la vinculación comunitaria? Pregunta necesaria que aparece en el proceso reflexivo y que nos lleva por una ruta de búsqueda. La Vinculación Comunitaria es la unión de dos términos igualmente sui generis, que es oportuno aclarar y comentar, nos referimos, primero, a la noción de Comunidad, que según algunos investigadores como Montero (2004), “...es un término polisémico, complejo y confuso...” es decir, encuentra varias aplicaciones según el tema en el que se encuentre involucrado. En tanto vinculación es definido por la RAE como la acción y efecto de vincular, a su vez definido cómo: (1) Atar o fundar algo en otra cosa, (2) Perpetuar o continuar algo o el ejercicio de ello, (3) Someter la suerte o el comportamiento de alguien o de algo a los de otra persona o cosa, (4) Sujetar a una obligación, (5) Sujetar o gravar los bienes a vínculo perpetuo y (6) Asegurar, atar con prisiones. Tenemos entonces una palabra que es utilizada para explicar diversas acciones, por lo que su uso depende del contexto donde la ubiquemos. 
Considerando nuestra experiencia en el ejercicio de la Vinculación Comunitaria, es factible considerar cuatro cuestiones básicas: (1) quien es el vinculador, (2) quien es el vinculado, (3) qué se quiere y para qué se quiere hacer vinculación, y (4) el sitio o destino de la vinculación. Ubicados bajo una ruta de acción, se reconoce una primer etapa con dos elementos importantes, el primero es la conexión entre el vinculante y el vinculado, para lo que se reconoce el concepto de 'primer contacto' (Freire, 1970), mismo que cobra importancia al facilitar el acceso (Hammersley y Atkenson, 2001) y/o introducción del vinculador a la comunidad que es sujeto de estudio, mismo que ha de influir en las futuras acciones que se seguirán, sobre todo con los actores sociales, con quienes se tendrá relación y en segundo término, a los vinculantes, en su caracterología y su manera de actuar en las relaciones interculturales.

La siguiente etapa del ejercicio implica la creación de vínculos de interés social y económico que se establecen en la acción, mediante el entretejimiento de intereses mutuos, pero también, y más importante aún, en la creación de lazos afectivos. Por lo tanto, hemos de considerar que este es un proceso vivencial, sentido, que sucede entre los vinculantes, y que construyen con la intención de establecer una relación de colaboración con objetivos claros, precisos y concretos.

En este momento el dialogo de saberes es esencial, pues ve más allá de la comunicación entre sujetos, ya que en ella se comprometen conocimientos, visiones, perspectivas y sentimientos diversos en una relación horizontal, de iguales, e implica el reconocimiento de esas diferencias (Peña, 2017). En suma, los objetivos del vinculador se conjugan con el del vinculante en una relación en que la colaboración es muy importante para que la relación sea efectiva y se logre exitosamente alcanzar lo que cada cual desea.

Cuando el proceso se ha alcanzado, se comienza una etapa de finalización, la cual puede ser a su vez el inicio de una etapa cualitativamente superior, tal cuestión depende del alcance de la investigación y de lo que la comunidad de estudio limite. La evaluación del proceso en estos momentos es clave para considerar los resultados y proponer, en su caso, nuevas acciones (Peña, 2017).

\section{LA INVESTIGACIÓN, EN LA FORMACIÓN DEL DESARROLLO SUSTENTABLE}

La investigación, a partir de contextos interculturales, tiene dos cuestiones esenciales que contribuyen a definir al profesional del Desarrollo Sustentable:

(1) En el ejercicio de la vinculación comunitaria, se induce al profesional en formación a usar el método científico, para hacer reconocimientos y análisis de las condiciones de vida de las comunidades sujeto de 
estudio, y sus vínculos con otras, en un intento por descubrir sus relaciones interculturales.

(2) Estos estudios, entre otras cuestiones, proponen tanto relacionar el concepto Sustentabilidad desde las comunidades de estudio, como medir el alcance de la sustentabilidad en dichas comunidades, apoyado, esto último, en las demandas de la sociedad surgidas ante las problemáticas ambientales, así como en el compromiso realizado por nuestro país en pro de la Sustentabilidad (pues ha suscrito diversos convenios internacionales, cuando menos a nivel institucional), lo cual supone que impactará en políticas públicas que se encaminan a lograr ese objetivo, por lo tanto, obliga a operar una investigación de tal complejidad.

En este punto es conveniente reafirmar que el término Comunidad es más apropiado como un concepto asociado al territorio, o espacio, ocupado por grupos humanos, ya que es el sitio que les da un sentido de pertenencia o "sentido de comunidad" (Montero, 2004), donde se interrelacionan o interactúan, comparten una cultura y una historia en común, que tiene un sentido dinámico y de cambio, en un sentido holístico.

De igual manera, el término desarrollo, asociado al mejoramiento de la calidad de vida de las personas, no se refiere a un crecimiento económico cuantitativo, sino más bien, como lo proponen Martínez y Figueroa (2014): “...al despliegue de potencialidades en un entorno de complejidad creciente... inmerso en...la búsqueda del bienestar colectivo e individual, a través del aprovechamiento de los bienes y servicios que oferta la naturaleza, en múltiples escalas espacio-temporales de los sistemas co-evolutivos".

Considerando lo escrito, es pertinente delinear las investigaciones a partir de tres ejes de análisis: (1) socio-cultural, (2) sistemas de producción, y (3) biodiversidad. Debería consistir en realizar una serie ordenada de estudios, bajo un enfoque mixto de investigación, con el fin de evaluar diversos componentes del sistema comunitario que ubiquen el desarrollo del sujeto de estudio desde una perspectiva sustentable (Sarandón y Flores, 2006), pero también desde sus relaciones interculturales.

Por último, estas investigaciones deberían tener en cuenta tres cuestiones: la primera es orientarse a realizar un reconocimiento o caracterización de las unidades de análisis, la segunda es analizar el progreso o evolución de las unidades de análisis hacia la sustentabilidad, considerando el uso de indicadores, así como de índices generales de sustentabilidad (Gravina y Leyva, 2012) y la tercera son los estudios de las relaciones comunitarias, que contribuyan a entender las relaciones dinámicas, estáticas e históricas de las comunidades sujeto de estudio, y por tanto, procura entender cómo interactúan, cómo se influyen, y que resultados, problemáticas y necesidades surgen. 


\section{CONCLUSIÓN}

Desde una mirada universitaria intercultural, la Sustentabilidad, más allá de ser un concepto a la vez polémico y esperanzador, es la parte medular en la búsqueda de un nuevo paradigma del desarrollo de las comunidades humanas y sus formas de relacionarse con su entorno, que se vive y se siente a partir de contextos culturales diversos en un territorio dado, por lo que las relaciones interculturales juegan un rol cada vez más preponderante, convirtiéndose en un elemento central para la formación de profesionales del Desarrollo Sustentable.

La confluencia del concepto Sustentabilidad, con otros dos igualmente poderosos como Desarrollo e Interculturalidad, son el sustento para la conformación de un profesional en el Desarrollo Sustentable, llamado a realizar estudios y análisis acerca de la relación de las comunidades humanas con su entorno, desde entender las relaciones históricas, dinámicas y estáticas que se establecen entre las diferentes culturas que comparten un mismo territorio, hasta reconocer cómo estas comunidades hacen uso y manejan sus recursos, bajo una lógica de medición del desarrollo sustentable alcanzado, cuyos efectos impactarán en la planificación que promueve alternativas de solución bajo principios éticos básicos, que contribuyen a una relación intercultural de armonía y convivencia que son el respeto, responsabilidad, cuidado y conocimientos.

Bajo estas premisas, la profesión en Desarrollo Sustentable sigue tanto a una investigación con enfoque mixto, que analice las situaciones en que los sujetos de estudio se encuentran y que reconozca qué tanto se encaminan a una vida sustentable, como del diagnóstico y diseño de soluciones, de la mano de un eje clave que es la vinculación comunitaria, debido a que sus acciones pueden modificar ideas preconcebidas por la gente, es decir, modificar pensamientos y actos de la gente, en el sentido de que se enfoquen hacia lo sustentable, en el contexto de la búsqueda de paradigmas como nuevas formas de relacionarse con el entorno.

Por lo tanto, el desarrollo y la interculturalidad desde la perspectiva de la sustentabilidad, son básicos para proponer políticas públicas que fortalezcan en las personas un espíritu de relación armoniosa con su entorno y promuevan, a su vez, niveles de calidad de vida sin destruir el mundo que nos rodea. 


\section{REFERENCIAS BIBLIOGRÁFICAS}

Albo X. S. (2003). Cultura, Interculturalidad Inculturación. Caracas: Ed. Federación Internacional de Fe y Alegría. IsBN: 980-313-017-X 70

Baronnet, B., y Bermúdez, M. (2019). La vinculación comunitaria en la formación de profesionales indígenas en México. Ciudad de México: Anuies. Dirección de producción editorial.

Bonilla, C. E., Hurtado P. J. y Jaramillo H. C. (2009). La investigación. Aproximaciones a la construcción del conocimiento científico. Colombia: Editorial Alfaomega.

Brundtland, G. H. (1987). Report of the World Commission on Environment and Development: Our common future (Reporte A/42/427). Recuperado de http://www. un-documents.net/wced-ocf.html

Casillas M. L. y L. Santini (2009). Universidad Intercultural Modelo educativo. México: Coord. General de Educación Intercultural y Bilingüe. SEP.

Freire, Paulo (1970). Pedagogía del oprimido. Montevideo: Tierra Nueva.

Fromm, E. (2006). El miedo a la Libertad. México D. F.: Ed. Paidós.

Gravina, H.B. y Leyva G.A. (2012). Utilización de nuevos índices para evaluar la sostenibilidad de un agroecosistema en la república bolivariana de Venezuela. Cultivos Tropicales. Vol. 33. No. 3 Pp. 15-22. Caracas.

Gunther D. (2009). Interculturalidad y diversidad cultural. Entrevista. Número 11 año 2. Revista Tukari. Guadalajara, Jalisco.

Hammersley M. y P. Atkenson (1994). Etnografía. Métodos de Investigación. El acceso. España: Ed. Paidós.

Harris, M. (1986). Caníbales y Reyes. Los orígenes de la cultura. Barcelona: Salvat editores. ISBN 84-345-8246-5.

Martínez, I., J., P. y Figueroa, C., A. (2014). Evolución de los conceptos y paradigmas que orientan la gestión ambiental ¿cuáles son sus limitaciones desde lo glocal? Revista Ingenierías vol. 13, No. 24. Colombia: Universidad de Medellín. IssN 1692 - 3324.

Montero, M. (2004). Introducción a la Psicología Comunitaria. Desarrollo, conceptos y procesos. Buenos Aires: Paidós.

Peña, P.J. (2017). La formación de profesionales en desarrollo sustentable en un programa de educación superior intercultural. CPU-e Revista de Investigación Educativa. Jalapa, Veracruz. ISSN 1870-5308.

Quijano, A. (2004). Colonialidad del poder, eurocentrismo y América Latina. En Edgardo Landler La Colonialidad del saber, eurocentrismo y ciencias sociales. Perspectivas latinoamericanas. Buenos Aires, Argentina: Clacso. Pp 231-246.

Sánchez Upegüi, A. (2010). Introducción: ¿qué es caracterizar? Medellín, Colombia: Fundación Universitaria Católica del Norte. 
Sarandón, J.S. y Flores C. (2009). Evaluación de la sustentabilidad en agroecosistemas: Una propuesta metodológica. Agroecología vol. 4. Buenos Aires, Argentina. Pp 19-28.

Universidad Intercultural de Chiapas (2013). Programa académico de la Licenciatura en Desarrollo Sustentable. Actualización. San Cristóbal de Las Casas, Chiapas: Academia de Desarrollo Sustentable-Universidad Intercultural de Chiapas.

Walsh, C. (2006). Interculturalidad y Colonialidad del poder: Un pensamiento y posicionamiento desde la diferencia colonial. En Interculturalidad, descolonización del estado y del conocimiento. Buenos Aires, Argentina: Ediciones del signo.

Walsh, C. (2009). Hacia una comprensión de la Interculturalidad. Revista Tukari. Año 2. Núm. 11. Guadalajara, Jalisco. Pp 6-7

Walsh, C. (2009). Interculturalidad crítica y Educación Intercultural. Ponencia del seminario Interculturalidad y Educación Intercultural. Instituto Internacional de Integración del Convenio Andrés Bello. La Paz, Bolivia. 